\title{
Performability Analysis for Feeding Unit of a Sugar Plant using Particle Swarm Optimization Technique
}

\author{
Gaurav Sharma* and P. C. Tewari \\ Department of Mechanical Engineering, N.I.T, Kurukshetra-136119, India
}

\begin{abstract}
The present study illustrates the application of the Particle Swarm Optimization technique in a sugar plant for the purpose enhancing the performance in terms of availability of the operational units. After evaluation and analysis of unit performance by applying Markov approach, the algorithm of PSO technique is applied to optimize the level of performance. The results of the work reflect the modification of solution using PSO and one can further receive the most advantageous blend of failure rates and repair rates that will be used further by the maintenance department for the design of effective maintenance strategies.
\end{abstract}

Keywords: performability analysis; feeding unit; markov technique; particle swarm optimization

(Submitted on November 5, 2019; Revised on November 30, 2019; Accepted on December 10, 2019)

(C) 2019 Totem Publisher, Inc. All rights reserved.

\section{Introduction}

In modern scenarios of automation, the high reliability of the industrial system is the main challenge for any industrial entrepreneur. The higher levels of unit availability result in performance enhancement as a whole for the plant. The availability level of the feeding unit has been optimised here in this work. There are a number of minor and major faults due where the feeding unit may undergo failure. Though failure cannot be totally avoided, by using optimal maintenance strategies, the maintenance department may ensure the maximum availability of units by keeping the units failure free for prolonged durations. Here, the Particle Swarm Optimization technique is proposed to optimize the level of performance of the feeding unit of the plant. Tan and Kramer [1] observed that the financial loss resulting from an unexpected shutdown may range from $\$ 500-\$ 100,000$ per hour. Haitang et al. [2] analysed the reliability of CAM on Gear Plate of D Type Knotter. Kumar and Tewari [3] conducted the optimization of a filling system at a beverage plant through the Particle Swarm Optimization technique. Kumar et al. [4] studied the impact of hot-cold redundancy of the pumps on the availability level of a thermal plant using the Markov technique. Lyonnet and Toscano [5] predicted the on-line reliability via a dynamic failure rate model. Wang et al. [6] evaluated the reliability of a multi-state series systems with performance sharing. Narahari and Vishwanadham [7] presented a Petri nets approach for modeling and analyzing flexible manufacturing systems (FMSs). Dekker and Groenendijk [8] discussed various availability assessment methods and their typical applications in industrial practices. Kumar et al. [9-10] used Markov modeling for analyzing and evaluating the performance of a repairable system of a sugar and fertilizer industry. Sachdeva et al. [11] performed a detailed analysis of a paper mill using Petri nets to evaluate the performance regarding the availability plant. Tavana [12] applied Petri nets for dynamic process modeling of an emergency management system at a nuclear power plant. Thangamani [13] dealt with the availability analysis of a lube oil system in a combined cycle power plant using a generalized stochastic Petri net, and the Monte Carlo simulation approach was used for further analysis. Singh and Garg [14] performed the availability analysis of plywood manufacturing systems under the assumption of exponential failure and repair rates. $\mathrm{Li}$ et al. [15] performed a modeling and availability analysis of navigation satellites based on colored Petri nets $(\mathrm{CPN})$ while considering both failures and performance status.

\footnotetext{
* Corresponding author.

E-mail address: reach4gaurav123@ rediff.com
} 


\section{Unit Description}

The feeding unit is the first unit of a sugar plant through which the cane enters the plant. There are a total five subsystems of the feeding unit and they are arranged in a systematic configuration.

- Subsystem A1: it has two cane unloaders in parallel. It is used to carry the cane from the trucks to the cane component lead to reduce capacity working.

- Subsystem A2: it includes one component of cane carrier and kicker in series configuration so as to feed the cane from the undloader to the cutter and leveler.

- Subsystem A3: A cane cutter component is dedicated to cutting the cane into small units.

- Subsystem A4: It consists of a fibrizor, which converts small pieces of cane into a pulp like mass.

- Subsystem A5: This subsystem covers one elevator and one conveyor. It transfers the pulp like mass of the cane to the roller mills.

\section{Assumptions and Notations}

\subsection{Assumptions}

Following are some assumptions employed for probabilistic modeling:

- The rates of failure and repair are invariable with time and are autonomous.

- A component will give a new performance for a specific time slot.

- Proper facilities for repair guarantee the immediate start of repair work after failure.

- Exponential distribution is followed by failure and repair rates of the components.

- Maintenance services include repair and/or replacement.

- Working of the unit in condition of reduced capacity is also possible.

\subsection{Notations}

Some of the notations used for performance analysis are as:

- The failure as well as repair rates stay invariable over time.

- A component after getting repaired works exactly the same as that of a new component.

- Reserve entities have same character and capability as that of active units.

- Service takes into account renovation and/or substitution.

- $\boldsymbol{O}, \square$ represent full capacity, reduced capacity and failed state respectively.

- $A_{1}, A_{2}, A_{3}, A_{4}$ and $A_{5}$ are operative states subsystems and $a_{1}, a_{2}, a_{3}, a_{4}$ and $a_{5}$ are failed states of subsystems.

- $\Phi_{1}, \Phi_{2}, \Phi_{3}, \Phi_{4}$ and $\Phi_{5}$ are failure rates of subsystems $A_{1}, A_{2}, A_{3}, A_{4}$ and $A_{5}$.

- $\mu_{1}, \mu_{2}, \mu_{3}, \mu_{4}$ and $\mu_{5}$ are repair rates of systems $A_{1}, A_{2}, A_{3}, A_{4}$ and $A_{5}$.

\section{Performance Modeling}

Performance modeling of the feeding unit is done by taking the exponential distribution of failure free time and the time involved in repair.

The 11 states associated with the above transition diagram (Figure 1) are as follows:

State 0 represents the operative state.

State 1 represents the reduced capacity state.

States 2, 3, 4, 5, 6, 7, 8, 9, 10 represent non-working or failed states.

$P_{0}(t)$ is the probability that the refining unit is working at full capacity, at any time $t$.

The following are the differential equations derived from the state transition diagram: 


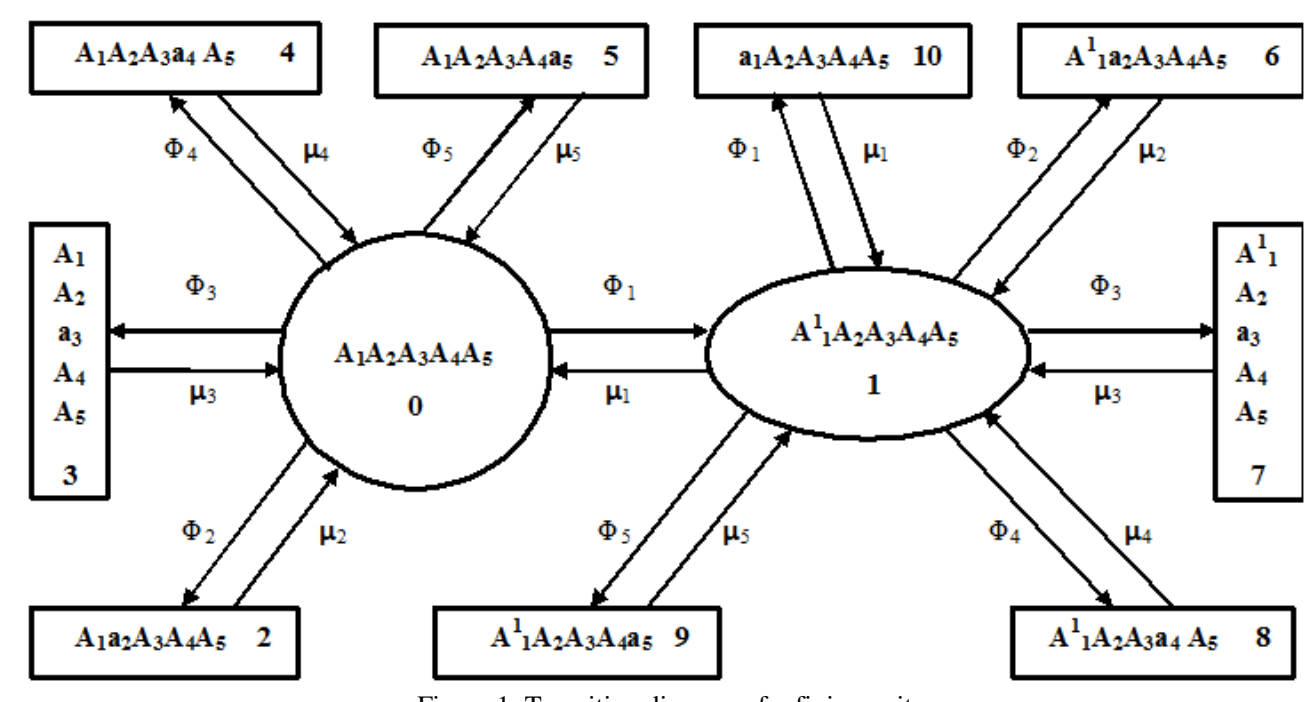

Figure 1. Transition diagram of refining unit

$$
\begin{aligned}
& P_{0}^{\prime}(t)+\Phi_{1} P_{0}(t)+ \Phi_{2} P_{0}(t)+\Phi_{3} P_{0}(t)+\Phi_{4} P_{0}(t)+\Phi_{5} P_{0}(t) \\
&= \mu_{1} P_{1}(t)+\mu_{2} P_{2}(t)+\mu_{3} P_{3}(t)+\mu_{4} P_{4}(t)+\mu_{5} P_{5}(t) \\
& P_{1}^{\prime}(t)+\Phi_{1} P_{1}(t)+\Phi_{2} P_{1}(t)+\Phi_{3} P_{1}(t)+\Phi_{4} P_{1}(t)+\Phi_{5} P_{1}(t)+\mu_{1} P_{1}(t) \\
&=\mu_{1} P_{10}(t)+\mu_{2} P_{6}(t)+\mu_{3} P_{7}(t)+\mu_{4} P_{8}(t)+\mu_{5} P_{9}(t)+\Phi_{1} P_{0}(t) \\
& P^{\prime}{ }_{2}(t)+\mu_{2} P_{2}(t)=\Phi_{2} P_{0}(t) \\
& P^{\prime}{ }_{3}(t)+\mu_{3} P_{3}(t)=\Phi_{3} P_{0}(t) \\
& P^{\prime}{ }_{4}(t)+\mu_{4} P_{4}(t)=\Phi_{4} P_{0}(t) \\
& P^{\prime}{ }_{5}(t)+\mu_{5} P_{5}(t)=\Phi_{5} P_{0}(t) \\
& P_{6}^{\prime}(t)+\mu_{2} P_{6}(t)=\Phi_{2} P_{1}(t) \\
& P^{\prime}{ }_{7}(t)+\mu_{3} P_{7}(t)=\Phi_{3} P_{1}(t) \\
& P^{\prime}{ }_{8}(t)+\mu_{4} P_{8}(t)=\Phi_{4} P_{1}(t) \\
& P_{9}^{\prime}(t)+\mu_{5} P_{9}(t)=\Phi_{5} P_{1}(t) \\
& P_{10}^{\prime}(t)+\mu_{1} P_{10}(t)=\Phi_{1} P_{1}(t)
\end{aligned}
$$

By means of Initial conditions at $t=0$,

$$
\begin{aligned}
& P_{i}(t)=1 \text { when } i=0 \\
& P_{i}(t)=0 \text { when } i \neq 0
\end{aligned}
$$

The availability under the steady state is calculated by placing $\mathrm{d} / \mathrm{d} t$ equal to 0 when $t$ approaches to $\infty$, which is shown in the above equations.

$$
\begin{gathered}
{\left[\Phi_{1}+\Phi_{2}+\Phi_{3}+\Phi_{4}+\Phi_{5}\right] P_{0}=\mu_{1} P_{1}+\mu_{2} P_{2}+\mu_{3} P_{3}+\mu_{4} P_{4}+\mu_{5} P_{5}} \\
{\left[\Phi_{1}+\Phi_{2}+\Phi_{3}+\Phi_{4}+\Phi_{5}+\mu_{1}\right] P_{1}=\mu_{1} P_{10}+\mu_{2} P_{6}+\mu_{3} P_{7}+\mu_{4} P_{8}+\mu_{5} P_{9}+\Phi_{1} P_{1}} \\
\mu_{2} P_{2}=\Phi_{2} P_{0} \\
\mu_{3} P_{3}=\Phi_{3} P_{0}
\end{gathered}
$$




$$
\begin{aligned}
& \mu_{4} P_{4}=\Phi_{4} P_{0} \\
& \mu_{5} P_{5}=\Phi_{5} P_{0} \\
& \mu_{2} P_{6}=\Phi_{2} P_{1} \\
& \mu_{3} P_{7}=\Phi_{3} P_{1} \\
& \mu_{4} P_{8}=\Phi_{4} P_{1} \\
& \mu_{5} P_{9}=\Phi_{5} P_{1} \\
& \mu_{1} P_{10}=\Phi_{1} P_{1}
\end{aligned}
$$

The full working probability of the unit i.e. $P_{0}$ is established by the applying the conditions of normalization.

$$
\sum_{i=0}^{10} P_{i}=1
$$

With substitution of values of $P_{1}$ to $P_{10}$ in form $P_{0}$, we get:

$$
\begin{gathered}
P_{0}+P_{1}+P_{2}+P_{3}+P_{4}+P_{5}+P_{6}+P_{7}+P_{8}+P_{9}+P_{10}=1 \\
P_{0}\left[1+G_{1}+G_{2}+G_{3}+G_{4}+G_{5}+G_{2} G_{1}+G_{3} G_{1}+G_{4} G_{1}+G_{5} G_{1}+G_{1} G_{1}\right]=1 \\
P_{0}\left[\left(1+G_{1}\right)+G_{2}\left(1+G_{1}\right)+G_{3}\left(1+G_{1}\right)+G_{4}\left(1+G_{1}\right)+G_{5}\left(1+G_{1}\right)+G_{1} G_{1}\right]=1 \\
P_{0}=1 /\left[\left(1+G_{1}\right)\left(1+G_{2}+G_{3}+G_{4}+G_{5}\right)+G_{1} G_{1}\right]
\end{gathered}
$$

The availability under the long run of the feeding unit may be attained by doing the precis of probabilities of existing states under full capacity and the states under reduced capacity.

$$
\begin{gathered}
A V_{1}=P_{0}+P_{1} \\
\text { Availability }=\left[1+G_{1}\right] /\left[\left(1+G_{1}\right)\left(1+G_{2}+G_{3}+G_{4}+G_{5}\right)+G_{1} G_{1}\right]
\end{gathered}
$$

Equation (23) above evaluates the performance of the feeding unit in terms of availability.

\section{Evolutionary Algorithm Particle Swarm Optimization}

The PSO technique is a technique inspired by the activities of bird people searching for food. At the start of the algorithm all ] birds are initialized in a random manner and everyone owns its position and velocity. It has been assumed that the ith particle owns the position and velocity as:

$$
\begin{aligned}
& X_{i}=\left(X_{i, 1}+X_{i, 2}+X_{i, 3}, \cdots, X_{i, n}\right) \\
& V_{i}=\left(V_{i, 1}+V_{i, 2}+V_{i, 3}, \cdots, V_{i, n}\right)
\end{aligned}
$$

Where, $n=$ population size.

The particles then start moving and with a change in position and velocity while trying to find the best solution in the search space. All the particles have the target to attain the best solution within the search space. The previously achieved best position of the particles and their neighbors are compared every time whenever a new position is achieved. After achieving the best position, the previous pbest and gbest are automatically updated. The algorithm will terminate in case they achieve the best solution or after execution of the maximum iterations. The algorithm of PSO is given in the figure. The flowchart demonstrating the basic PSO algorithm has been given by Figure 2. The expressions given below give the updated velocity and position, respectively, of the particle. 


$$
\begin{gathered}
V_{i}=w \times V_{i}+c_{1} \times \text { rand }_{1} \times\left(\text { pbest }_{i}-X_{i}\right)+c_{2} \times \text { rand }_{2} \times(\text { gbest }) \times X_{i} \\
X_{i}=V_{i}+X_{i}
\end{gathered}
$$

$$
\begin{gathered}
\left.c_{1}=\text { cognitive component (range } 0 \text { to } 2\right) \\
\left.c_{2}=\text { cognitive component (range } 0 \text { to } 2\right) \\
\left.\operatorname{rand}_{1}=\text { random number ( } 0 \text { to } 1\right) \\
\left.\operatorname{rand}_{2}=\text { random number ( } 0 \text { to } 2\right) \\
w=\text { inertia weight }(0.4 \text { to } 1.9)
\end{gathered}
$$

The inertia weight has the ability to control the global (with higher value) as well as local (with lower value) search in the overall search space. Inertia weight is updated by the relation given below:

$$
\begin{gathered}
w=\text { wmax }-(\text { wmax }- \text { wmin }) \times \text { ite } / \text { maxite } \\
\text { where ite }=\text { iteration number } \\
\text { maxite }=\text { maximum iterations }
\end{gathered}
$$$$
\text { wmax and wmin = initial value and the final valve }
$$

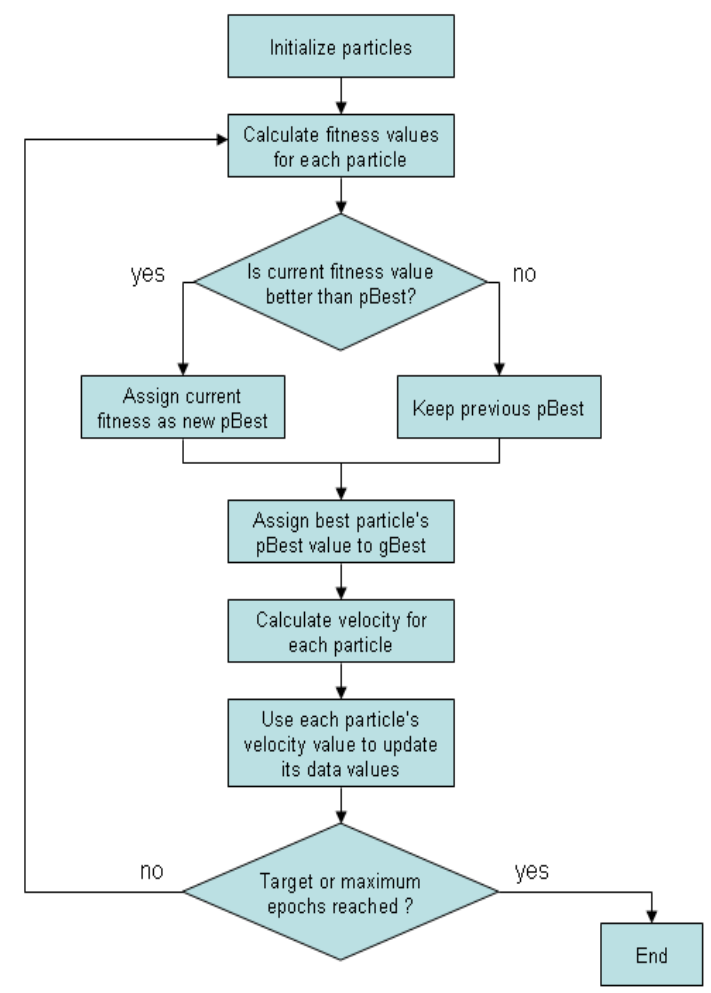

Figure 2. PSO algorithm

\section{Performability Optimization}

After carrying out the performance analysis using the Markov approach, it has been found that the maximum performance level of the unit in terms of availability comes out to be $81.15 \%$. Particle Swarm Optimization is then used in order to optimize the performability level of the feeding unit. The comparison of the availability level obtained by the Markov technique and PSO technique has been done. Table 1 shows the parameters that are needed to use PSO. Optimization of the 
unit performance is done by varying the population (particle) size first and then the number of generations. Performance of the unit changes by the above variation. Table 2 and Table 3 reflect how the performance of the unit changes and gets optimized with the variation in population size and also with the change in number of generations. The ranges designed for failure rates and repair rates parameters of different subsystems are as:

$$
\begin{gathered}
\Phi_{1}=(0.01,0.09), \mu_{1}=(0.15,0.35), \Phi_{2}=(0.01,0.05), \mu_{2}=(0.05,0.25) \\
\Phi_{3}=(0.00062,0.00070), \mu_{3}=(0.03,0.07), \Phi_{4}=(0.0004,0.0008), \mu_{4}=(0.015,0.075) \\
\Phi_{5}=(0.015,0.035), \mu_{5}=(0.10,1.30)
\end{gathered}
$$

Table 1. Parameters for PSO

\begin{tabular}{|c|l|c|c|}
\hline Sr. No. & \multicolumn{1}{|c|}{ Parameters } & Values & Remarks \\
\hline 1 & Inertia weight $(w)$ & 0.4 to 0.9 & Generally lies between $0-1$ \\
\hline 2 & Cognitive component $\left(c_{1}\right)$ & $0-2$ & Selected Randomly $(0-2)$ \\
\hline 3 & Cognitive component $\left(c_{2}\right)$ & $0-2$ & Selected Randomly $(0-2)$ \\
\hline 4 & Population size & 20 to 100 & 20 to 100 with step size 10 \\
\hline 5 & Number of generations & 20 to 100 & 20 to 100 with step size 10 \\
\hline
\end{tabular}

Table 2. Trend of availability of feeding unit with number of generations using PSO

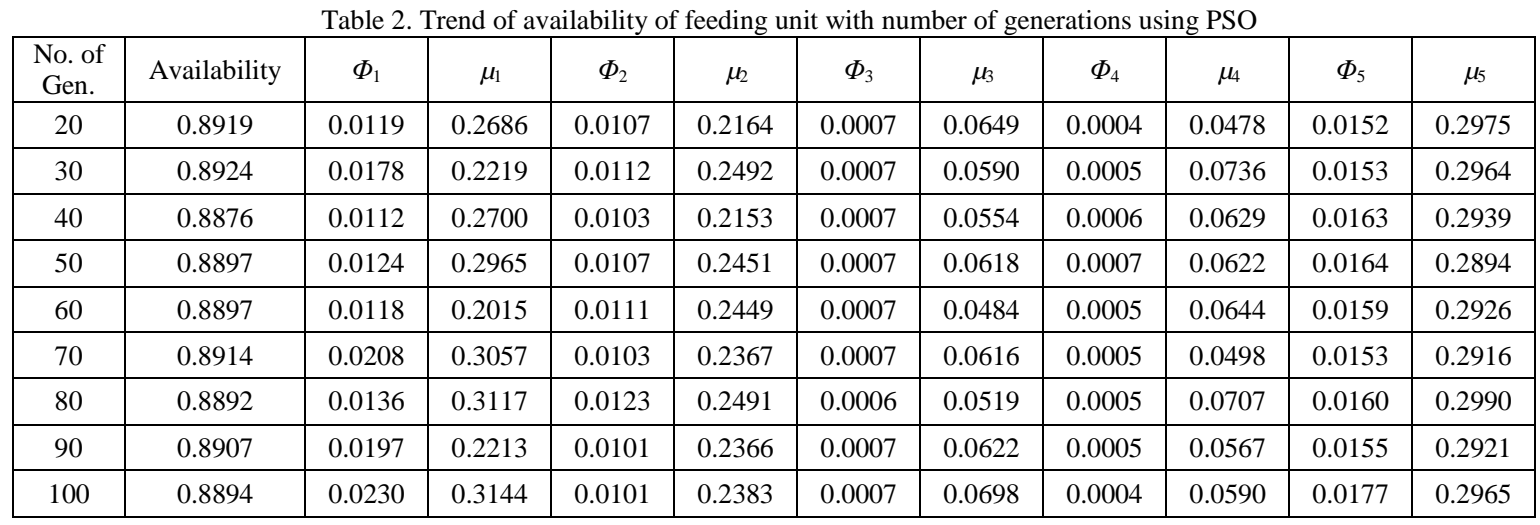

\begin{tabular}{|c|c|c|c|c|c|c|c|c|c|c|c|}
\hline $\begin{array}{c}\text { Pop. } \\
\text { Size }\end{array}$ & Availability & $\Phi_{1}$ & $\mu_{1}$ & $\Phi_{2}$ & $\mu_{2}$ & $\Phi_{3}$ & $\mu_{3}$ & $\Phi_{4}$ & $\mu_{4}$ & $\Phi_{5}$ & $\mu_{5}$ \\
\hline 20 & 0.8868 & 0.0166 & 0.3273 & 0.0107 & 0.2434 & 0.0007 & 0.0559 & 0.0004 & 0.0336 & 0.0164 & 0.2922 \\
\hline 30 & 0.8899 & 0.0165 & 0.2335 & 0.0101 & 0.2419 & 0.0007 & 0.0634 & 0.0005 & 0.0742 & 0.0175 & 0.2904 \\
\hline 40 & 0.8911 & 0.0160 & 0.3358 & 0.0113 & 0.2487 & 0.0007 & 0.0695 & 0.0005 & 0.0539 & 0.0152 & 0.2704 \\
\hline 50 & 0.8896 & 0.0147 & 0.2527 & 0.0101 & 0.2074 & 0.0006 & 0.0612 & 0.0005 & 0.0545 & 0.0154 & 0.2914 \\
\hline 60 & 0.8922 & 0.0104 & 0.3125 & 0.0105 & 0.2172 & 0.0006 & 0.0629 & 0.0004 & 0.0633 & 0.0164 & 0.2999 \\
\hline 70 & 0.8918 & 0.0100 & 0.1770 & 0.0108 & 0.2355 & 0.0007 & 0.0618 & 0.0004 & 0.0664 & 0.0150 & 0.2714 \\
\hline 80 & 0.8920 & 0.0143 & 0.3285 & 0.0102 & 0.2461 & 0.0007 & 0.0578 & 0.0005 & 0.0747 & 0.0168 & 0.2826 \\
\hline 90 & 0.8891 & 0.0148 & 0.2073 & 0.0113 & 0.2385 & 0.0007 & 0.0609 & 0.0005 & 0.0481 & 0.0153 & 0.2959 \\
\hline 100 & 0.8878 & 0.0118 & 0.2142 & 0.0109 & 0.2415 & 0.0007 & 0.0587 & 0.0007 & 0.0687 & 0.0151 & 0.2684 \\
\hline
\end{tabular}

A simulation has been done by making variations in the number of generations from 10 to 100 with 10 as the step size. The population is kept at 80 as a constant. The impact of this can be seen through Figure 3.

The performance of the feeding unit is calculated as 89.24 by PSO, which shows that there is a clear rise in the level of availability by $8 \%$. The optimum combination of failure rates and repair rates is observed at 30 number of generations, $\Phi_{1}=0.0178, \mu_{1}=0.2219, \Phi_{2}=0.0112, \mu_{2}=0.2492, \Phi_{3}=0.0007, \mu_{3}=0.0590, \Phi_{4}=0.0005, \mu_{4}=0.0736, \Phi_{5}=0.0153$ and $\mu_{5}=1.2964$. (Table 2)

Similarly, in the next simulation, the number of generations is kept constant (i.e. 80) and this time the population size varied from 20 to 100. The impact of this can be seen through Figure 4. 


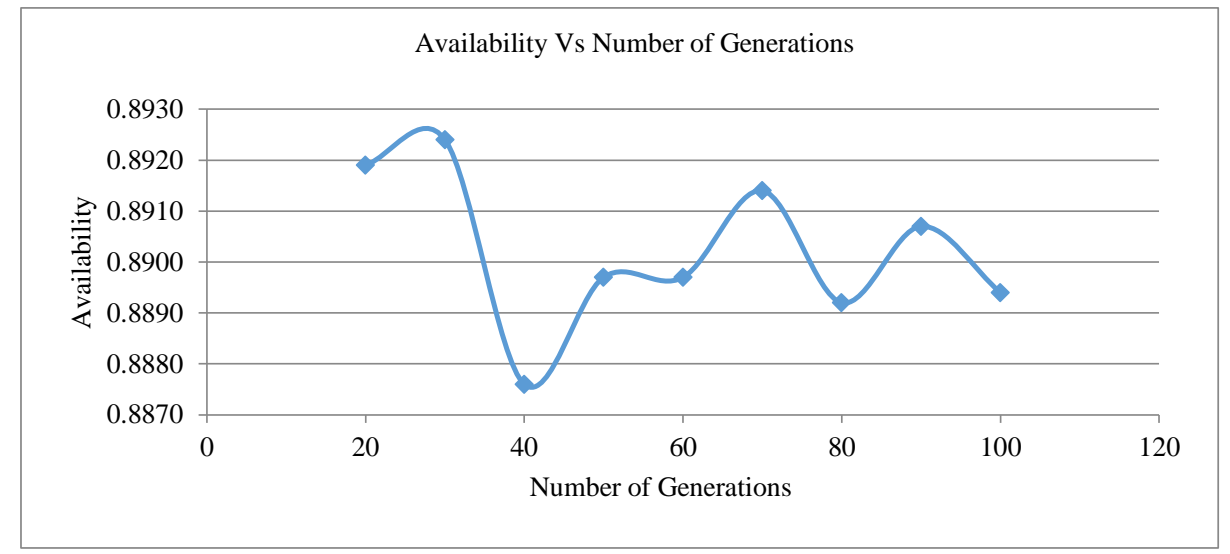

Figure 3. Trend of availability with number of generations

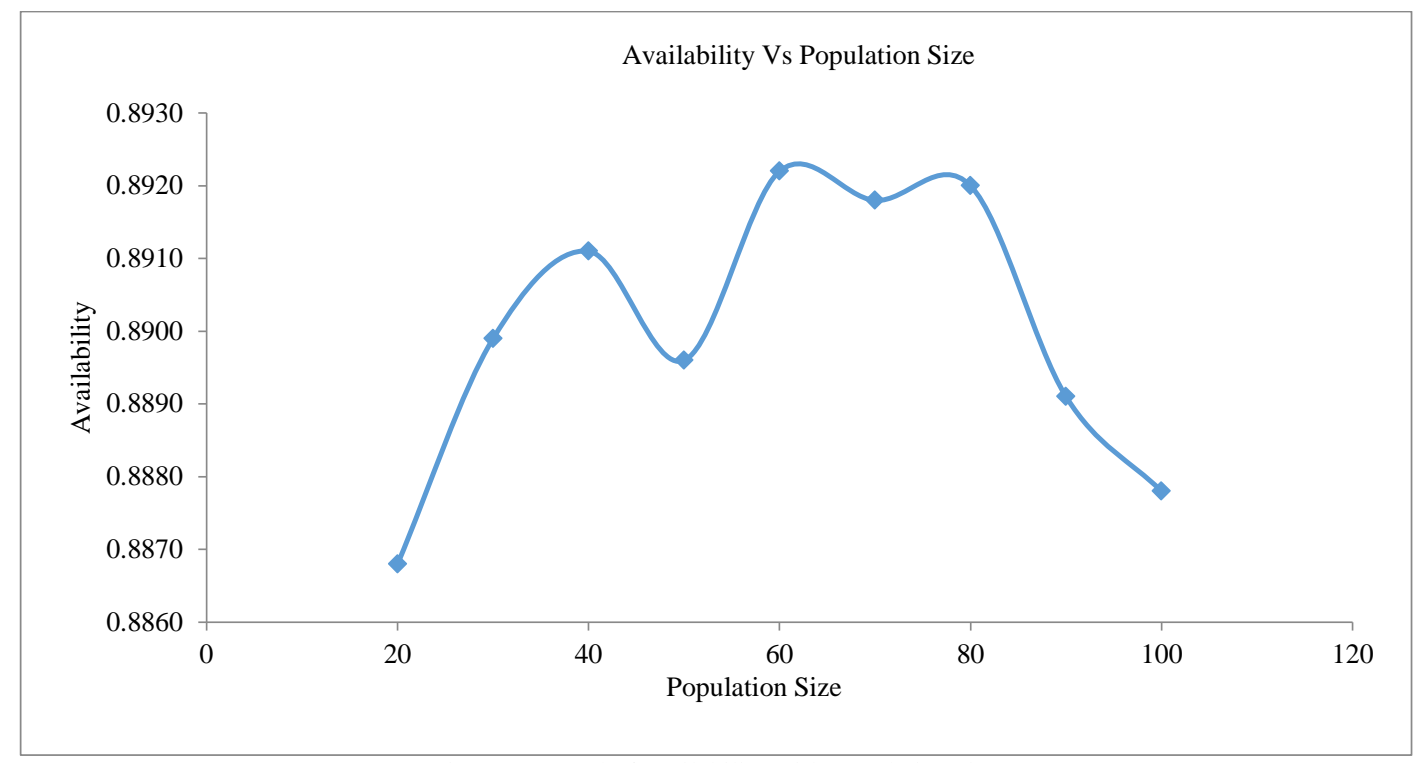

Figure 4. Trend of availability with population size

The performance level gets enhanced to $89.22 \%$ using PSO, which imparts fine blends of failure rate and repair rate parameters as $\Phi_{1}=0.0104, \mu_{1}=0.3125, \Phi_{2}=0.0105, \mu_{2}=0.2172, \Phi_{3}=0.0006, \mu_{3}=0.0629, \Phi_{4}=0.0004, \mu_{4}=0.0633, \Phi_{5}=$ $0.0164, \mu_{5}=1.29994$, at 80 number of generations (Table 3).

\section{Conclusions}

The research work helps clear the understanding of the behavior or the feeding unit in actual practical environments. The proper scheduling of each process and activity is very necessary in such an environment. This work is done to optimize the performance of the feeding unit in terms of availability. Further, the work assists in designing the appropriate policy for maintenance work on the plant. Random failures can then be very easily handled and idle times for the machines can be reduced to minimum possible levels. In short, the results highlight the optimized performance level of the feeding unit in sugar plants.

\section{References}

1. K J. S. Tan and M. A. Kramer, "A General Framework for Preventive Maintenance Optimization in Chemical Process operations," Computers and Chemical Engineering, Vol. 21, pp. 1451-1469, December 1997

2. C. Haitang, L. Peiwen, and S. Xueke, "Reliability Analysis of the CAM on the Drive Gear Plate of D Type Knotter," International Journal of Performability Engineering, Vol. 15, No. 1, pp. 23-34, January 2019

3. P. Kumar and P. C. Tewari, "Performance Analysis and Optimization for CSDGB Filling System of a Beverage Plant using Particle Swarm Optimization," International Journal of Industrial Engineering Computations, Vol. 8, No. 3, pp. 303-314, January 2017

4. R. Kumar, A. Sharma, and P. C. Tewari, "Effect of Various Pump Hot-Cold Redundancy on Availability of Thermal Power 
Plant Subsystems," International Journal of Intelligent Enterprise, Vol. 2, No. 4, pp. 311-324, April 2014

5. P. Lyonnet and R. Toscano, "On-Line Reliability Prediction via Dynamic Failure Rate Model," IEEE Transactions on Reliability, Vol. 57, No. 3, pp. 452-457, September 2008

6. G. Wang, F. Duan, and Y. Zhou, "Reliability Evaluation of Multi-State Series Systems with Performance Sharing," Reliability Engineering and System Safety, Vol. 173, No. 3, pp. 58-63, May 2018

7. Y. Narahari and N. Vishwanadham, "A Petri Net Approach to the Modeling and Analysis of Flexible Manufacturing Systems," Annals of Operations Research, Vol. 3, No. 8, pp. 449-472, August 1985

8. R. Dekker and W. Groenendijk, "Availability Assessment Methods and Their Application in Practice," Microelectronics Reliability, Vol. 35, No. 9-10, pp. 1257-1274, September 1995

9. D. Kumar, J. Singh, and P. C. Pandey, "Availability Analysis of Crystallization System in the Sugar Industry under Common Cause Failure," IEEE Transactions on Reliability, Vol. 41, No. 1, pp. 85-91, October 1992

10. D. Kumar, P. C. Pandey, and J. Singh, "Process Design for a Crystallization System in the Urea Fertilizer Industry," Microelectronics Reliability, Vol. 31, pp. 855-859, May 1991

11. A. Sachdeva, P. Kumar, and D. Kumar, "Behavioral and Performance Analysis of Feeding System using Stochastic Reward Nets," International Journal of Advanced Manufacturing Technology, Vol. 45, pp. 156-169, February 2009

12. M. Tavana, "Dynamic Process Modeling using Petri Nets with Applications to Nuclear Power Plant Emergency Management," International Journal of Simulation and Process Modeling, Vol. 4, No. 2, pp. 130-138, June 2008

13. G. Thangamani, "Generalized Stochastic Petri Nets for Reliability Analysis of Lube Oil System with Common-Cause Failures," American Journal of Computational and Applied Mathematics, Vol. 2, No. 4, pp. 152-158, August 2012

14. J. Singh and S. Garg, "Availability Analysis of Core Veneer Manufacturing System in Plywood Industry," in Proceedings of International Conference on Reliability and Safety Engineering, pp. 497-508, June 2005

15. H. S. Li, H. Zheng, H. T. Zhao, and Z. X. Zheng, "Research on the Availability Analysis Method of Navigation Satellite based on Petri Nets," in Proceedings of China Satellite Navigation Conference, pp. 127-136, May 2018

Gaurav Sharma is a Ph.D. research scholar in the Department of Mechanical Engineering at the National Institute of Technology, Kurukshetra, India. His research areas of interest include Reliability, Simulation Modeling, Performance Optimization and System Behavioral Analysis.

P. C. Tewari is a regular Professor in the Department of Mechanical Engineering at the National Institute of Technology, Kurukshetra, India. His fields of specializations are Industrial Engineering, Mathematical Modeling, Performance Evaluation and Optimization, System Behavioral Analysis, Reliability and Maintenance Resource Allocation. 